\title{
Individual differences in speech fusion: Methodological and theoretical explorations
}

\author{
STEVEN W. KEELE \\ University of Oregon, Eugene, Oregon \\ and \\ DON R. LYON \\ University of Dayton Research Institute, Dayton, Ohio
}

\begin{abstract}
Day (Note 1) constructed pairs of speech sounds such as "lanket" and "banket" from words (e.g., blanket) by omitting either the first or second consonant. When these two components were presented about the same time, one to each ear, some people appeared to fuse them, reporting they heard the word. Other people rarely fused. Moreover, if asked to report the first letter they heard, a judgment of temporal order, subjects that tended to fuse also reported what would be the first consonant of the word, even when it was not first in the pair of components. From this and other evidence, Day concluded that the perception of some individuals might be "language-bound." We tested a strong version of the language-boundness view of fusion and were able to reject it. We also examined several other possible causes of individual differences in fusion rate. Our second experiment provides evidence that a more general failure to discriminate temporal order of even nonlinguistic material may be involved.
\end{abstract}

This study investigates a striking individual difference in perception reported by Day (Note 1). She presented people with pairs of tape-recorded speech components constructed from words. The words were all ones in which a stop consonant (b, p, d, t, g, or k) was followed by a liquid consonant ( $\mathrm{r}$ or 1 ), such as "blanket," "closet," and "greedy." The components were derived from a word by omitting either the initial stop or liquid consonant during pronunciation, producing components such as "banket-lanket," "coset-loset," and "geedyreedy." When a pair was presented, one component to each ear at about the same time, many people reported hearing a word despite the fact that no word was present on the recording. However, large individual differences appeared. While some people fused more than half the pairs, other people rarely fused them, and the result was a marked bimodal distribution of number of people plotted against fusion rate.

The individual variation in fusion generated much interest, partly because it appeared so marked and partly because it appeared to reflect very basic differences in

We would like to thank Barbara Lewis, Suzanne de Lemos, Elizabeth Rodriguez, and David Bachman for their contributions to this project. Funding was supplied by the Office of Naval Research Contracts N00014-77-C-0643 and N00014-76-C-0344. S. W. Keele's complete mailing address is: Department of Psychology, University of Oregon, Eugene, Oregon 97403; D. R. Lyon's complete mailing address is: University of Dayton Research Institute, Dayton, Ohio 45409. cognitive function. Day (Note 2, Note 3) found, for example, that fusers tend to have shorter digit spans than nonfusers and are less adept than nonfusers at a language game in which the $r$ letters in words were to be pronounced as 1 and the 1 letters pronounced as $r$. Fusers, in Day's terms, appear more bound to normal language.

Subsequent experiments by Poltrock and Hunt (1977) failed to replicate the strong bimodality of individual differences in fusion that Day had found. However, Poltrock and Hunt concluded that their results were "consistent with the hypothesis that individual differences in fusion rate are due to differences in perceptual dependence on linguistic rules" (1977, p. 62). This is a weaker hypothesis than Day's, but it is still an important one. Unfortunately, as Poltrock and Hunt themselves note (p. 73), their experiments provide no direct test of this hypothesis.

The purpose of the present paper is to examine this hypothesis of linguistic dependence in detail. In doing this, we report two experiments. The first one is concerned with examining the many potential ways of measuring individual differences in fusion to see which ones best capture the phenomenon. This experiment is reported here because the various potential measures of fusion turn out to have surprisingly little individual differences variance in common. We view this as a serious problem with Day's original view of fusion.

Our second experiment looks at various more detailed versions of the general notion that individual differences 
in fusion rate are related to linguistic effects on perception. We present several possible processing mechanisms by which such effects could operate and some relevant experimental evidence, most of which is negative. Finally, we present evidence that suggests more directly that individual differences in fusion may have less to do with linguistic processes than with the ability to correctly perceive the temporal order of auditory events.

\section{EXPERIMENT 1: METHODOLOGICAL ISSUES OF FUSION}

One methodological question examined in past studies is, what physical variables influence fusion? Day (Note 1) found that whether the onset of the consonant led the liquid by up to $100 \mathrm{msec}$ or whether the liquid led the stop by up to $100 \mathrm{msec}$, temporal asynchrony had little influence on the proportion of times the components fused. Poltrock and Hunt (1977) generally replicated this result: The value of temporal asynchrony had little effect on fusion rate, except that fusion was more frequent when the stop led the liquid. Other physical variables have remarkably little effect on fusion. Intensity differences of as much as $15 \mathrm{~dB}$ between the components, fundamental frequency differences of $20 \mathrm{~Hz}$, simulated difference in vocal tract size, and differences on all three dimensions had virtually no effect on fusion rates (Cutting, 1975).

The relative lack of influence of physical variables on fusion suggests that fusion is a central phenomenon, and it has the practical implication that minor differences in the components when constructing natural language tapes, as in the present study, are probably of little consequence.

A second methodological question, and one on which our study focuses, concerns how different measures of fusion relate to each other. A number of methods of eliciting and detecting fusions have been proposed in earlier studies. An initial procedure used by Day required people to write down what they heard, whether one or two items and whether nonsense or real words. This procedure, when applied to individual differences, is highly susceptible to response bias; that is, it may be highly influenced by the subject's expectations about whether or not words are really recorded. In fact, since there are no words, there is no way of knowing whether subjects would be able to discriminate fused words from real words, if given an opportunity. In signal detection terms, there is a failure to differentiate bias and discriminability. An understanding of the fusion phenomenon would be fostered by separating the two aspects of performance.

Another technique explored by Day asked people to judge the first letter in an utterance (for example, in the LANKET-BANKET pair, subjects indicated whether they heard an " 1 " or a " $b$ " first). Again, there was a large range of individual differences. Day mea- sured the percent correct judgments when the liquid consonant led the stop. Some people exhibited a high percent correct on that measure, and some exhibited a very low percent correct. Percent correct on the liquidled pairs was negatively correlated with fusion scores, but the correlation was not unity and, in fact, was neither reported nor, apparently, statistically validated. Moreover, the temporal order judgment scores, although exhibiting a broad range, were not as noticeably bimodal as the fusion scores.

Measuring only percent correct on the liquid-led pairs again confounds discriminability and bias. A person with an extreme bias to report the stop to lead might almost always be correct when the stop actually leads and slightly less than $50 \%$ correct on liquidleading pairs. Such a pattern of results would actually indicate considerable discriminability if the effect of bias were removed. One measure of discriminability that is fairly free of bias is the signal detection measure of $\mathrm{d}^{+}$; another is the percent correct averaged over both stopled and liquid-led pairs. Both of these measures are used in the present study.

Independently of our own study, Poltrock and Hunt (1977) compared a bias measure, percent correct on stop leads minus percent correct on liquid leads, and a discriminability measure of overall percent correct with fusion rates. The bias measure correlated significantly with fusion rates $(r=.48)$, but the discriminability measure did not $(\mathrm{r}=.21)$.

In considering these different correlates of fusion, it is clear that the concept itself can have several distinct meanings. In the strongest sense of the term, the occurrence of a fusion would necessarily prohibit the subject's reporting the perceptual details that define the two separate components. Thus, on a trial in which fusion occurs, the subject should be unable to discriminate which component was presented first and which component was presented to which ear. If, on a given trial, a subject reports veridically either (1) that the liquid led or (2) that the liquid was presented to a particular ear and the stop was presented to the other, then by the strong definition a fusion could not have taken place on that trial.

Our first experiment tests some predictions of this strong view of fusion as a source of individual differences. We believe that this view corresponds best with Day's original concept. Indeed, it is the basis of her linking of performance on the temporal order judgment task to subjective reports of fusions. If each time an individual hears a genuine fusion, he/she fails to hear the two components, that should preclude any accurate judgment that depends on hearing the two components separately.

In this experiment, three such judgment tasks were explored. One task involved temporal order judgment of the components (e.g., LANKET or BANKET). A second task asked subjects to selectively report the components 
in the right ear. Subjects that fuse the items from the two ears should have difficulty with both of these judgments. The third task involved word-nonword judgments. On half of the trials, an actual word was played in both ears. On the other half of the trials, only the component pairs were presented. Subjects were asked to judge whether a real word was present. If people actually fuse the components from the two ears, the three objective measures should correlate with each other.

\section{Method}

Subjects. Thirty-six native speakers of English participated for two sessions. They were paid $\$ 4$ plus a bonus for accurate performance. All subjects claimed to be right-handed and wrote with their right hands. In most cases, subjects were run four at a time, each with a separate set of earphones and with partitions to prevent subjects' viewing each other.

Materials. The stimuli were tape recordings of 22 different words and components derived from the words; the words are shown in Table 1. Each word started with one of the stop consonants $\mathrm{p}, \mathrm{t}, \mathrm{k}, \mathrm{b}, \mathrm{d}$, or $\mathrm{g}$, and the second letter of a word was the liquid $r$ or 1 . The components of a word were constructed by the speaker's eliminating either the first or second letter as he pronounced the word. For example, the word TRUMPET yielded two components, TUMPET and RUMPET. A professional speaker, a former radio announcer, prepared a master tape of the words and the components. When the master tape was slowly moved by hand back and forth over the playback head of the recorder, it was usually possible to localize the onset of the word or component within a few milliseconds. However, on some of the recordings, the onset of speech sound was so gradual that it was difficult to specify when the onset began. In addition, there were occasional noise disturbances near the beginning of the speech sounds. These two problems were corrected by selectively erasing the noise disturbances and the earliest portion of the speech sound to give a sharper onset. Individual pronunciations following the editing were clearly discriminable.

A copy of the master tape was made, and the onsets of the speech sounds on both tapes were again determined by passing the tape over the playback head by hand. The onsets were visually marked. The two tapes were then mounted on a pair of Ampex playback units owned by the University of Oregon Audio-Visual Department and wired for simultaneous starting. The visual marks on the two master tapes could be aligned with reference marks on the playback units, both units simultaneously started, and the messages transferred to two channels of a third recorder. This procedure allowed the components on the two channels to be aligned within approximately $\pm 10 \mathrm{msec}$ of the desired spacing.

Three different experimental tapes were constructed. On one tape, intended for temporal order judgments, pairs of com-

Table 1

Wonds Used for Constructing Components

\begin{tabular}{ll} 
BLANKET & GLADLY \\
BLOSSOM & GLEAMING \\
BRACELET & GRAVITY \\
BREAKFAST & GREEDY \\
CLIMATE & PLACID \\
CLOSET & PLANET \\
CRACKER & PRIVATE \\
CRYSTAL & PRODUCT \\
DRAGONS & TREATMENT \\
DREADFUL & TRIUMPH \\
DROWSY & TRUMPET \\
\hline
\end{tabular}

ponents from the same word (e.g., PANET-LANET) were recorded, one component on each channel. The start of one component led the other by $80 \mathrm{msec}$. As a consequence, the remaining phonemes in the pairs also tended to be out of synchrony. Each of the 22 component pairs was recorded twice, for a list length of 44 . On half of the pairs, the stop consonant led the liquid, and on the other half, the liquid led the stop. The stop-led and liquid-led pairs were randomly mixed in order on the tape, and 7-8 sec intervened between successive pairs.

A second tape to be used for right-ear judgments also was constructed from the component pairs, but in this case the onset times were approximately synchronized. An equal number of stop-consonant and liquid-consonant components occurred in the right ear, and these two types were randomly intermixed in the list. Again, the list was composed of 44 pairs, with about $7-8$ sec between pairs.

A third tape was designed for word judgments. Half of the 44 pairs in the list were component pairs as before, but with the stop component always leading the liquid component by approximately $80 \mathrm{msec}$. For the remaining 22 pairs, the same word (e.g., TRIUMPH and TRIUMPH) was presented to both ears. To prevent discrimination's being based on extraneous cues, the word in one ear led the word in the other ear by approximately $80 \mathrm{msec}$ and the two recordings of the word were independent pronunciations.

Checklists for the subjects were constructed for each tape. The list for the temporal order judgment and the right-ear judgment tapes listed the 44 component pairs. The list for the word judgment task listed the word and the component pair for each of the 44 list positions. Thus, in all conditions, the subjects were aware in advance of the possible identities of the items.

Procedure. The subjects listened to each of the three tape recordings of 44 pairs on each of two successive days. The order of presentation was: temporal order judgment, right-ear judgment, word judgment. The session on each day lasted about $30 \mathrm{~min}$. Thus, over the two sessions, each subject made $88 \mathrm{judg}-$ ments in each of the three conditions.

For the temporal order judgment, it was explained that no words were actually recorded, even though it might on occasion sound as though a word was present. They were to listen to a component pair and then check on the list before them which component they thought began first. Subjects were asked to fix their eyes straight ahead.

On the right-ear judgment task, subjects were again informed of the nature of the items and were then told to listen only to their right ear, checking the component on the list that occurred on that ear. Gopher (1973) noted that when subjects attend to only one side in dichotic listening, most of them also turn their eyes in that direction. To avoid any possible influence of individual difference in eye positioning on individual differences in reporting items on the right ear, all subjects in this experiment were asked in the right-ear judgment task to turn their eyes to the right.

On the word judgment task, subjects were informed that on half the trials there actually would be the same word on both ears and on the other half there would only be components.

Pilot testing had indicated that, at least on temporal order and right-ear judgments, some people perform at near-chance levels. As an incentive for people to concentrate on the task despite difficult discriminations, they were paid .75 cents for each correct response, yielding a bonus of up to $\$ 1.96$ in addition to their normal pay.

\section{Results}

The distribution of subjects by total percent error is shown in Figure 1. On both temporal order judgments and ear judgments, people exhibit wide individual differences, ranging from about $10 \%$ error to chance 

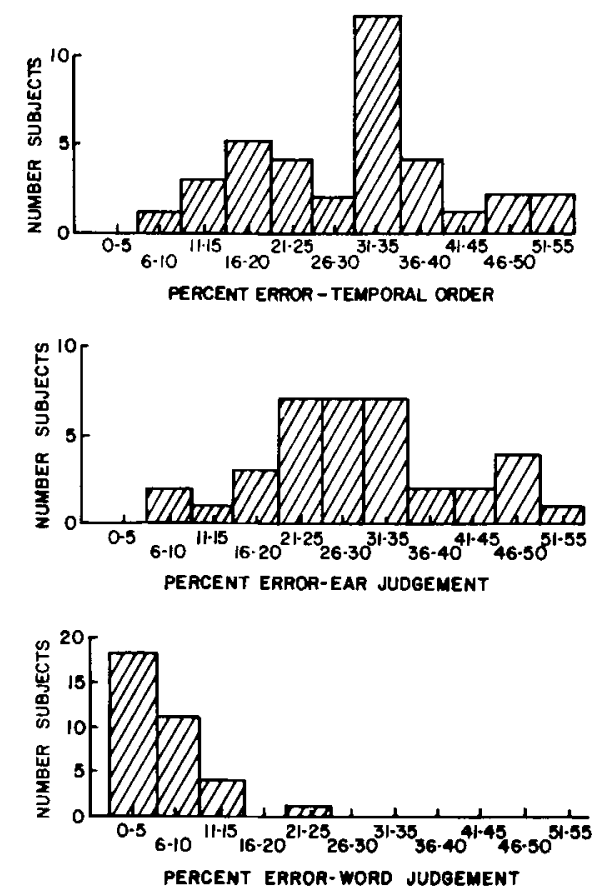

Figure 1. Number of subjects in each task that show a specific error percentage.

levels of $50 \%$ error. On the word judgment task, the range of scores is much more restricted and people are more accurate. Some of our subjects made no errors at all, and half made 5\% or fewer errors. The latter task indicates either that pure fusion was rather rare for most subjects or that there were enough residual cues present to allow most subjects to discriminate the fused product from a real word.

Subjects appear to have been continuously distributed in performance on each of the tasks, and there was little evidence of bimodality in any of the distributions, as one might expect from Day's (Note 1) data. A continuous distribution could have occurred if abilities on the tasks had been truly discontinuous, but the assessment was unreliable. Since each task was presented on two sessions, performance on each task was correlated across 2 days. The correlations were .65 for temporal order judgment, .81 for ear judgment, and .65 for word judgment. Applying the Spearman-Brown formula for reliability of the score totaled over both days (Guilford, 1954), the test reliabilities were .79 for temporal order judgment, .89 for ear judgment, and .79 for word judgment. Since the tests were fairly reliable, it appears that the distribution of errors on the tasks were not bimodal.

The Pearson correlation between errors on temporal order judgment and errors on ear judgment was .38; between temporal order and word judgment, it was .23; and between ear judgment and word judgment, it was .24. Only the first correlation was significant, at the .025 level of confidence.
If subjects tend to perceptually fuse the components into a word, then there might be a bias on the temporal order judgment to say that the stop consonant leads the liquid. Thus errors on that task may be a combination of difficulty in discriminating and response bias. To obtain a more pure measure of discriminability, the temporal order errors were broken down into cases in which stop-led stimuli were incorrectly identified as liquid leads and vice versa, and the statistic d' was calculated for each subject as a measure of perceptual discriminability between the two classes. This more refined measure had little influence on the results: $\mathrm{d}^{\prime}$ correlated .97 with total percent correct, indicating little difference in the two measures; $\mathrm{d}^{\prime}$ correlated -.31 with ear judgment and -.27 with word judgment, with only the former being significant at the .05 level of confidence.

The highest correlation, therefore, was between errors on temporal order judgment and errors on the ear judgment; its magnitude was .38. The correlation presumably would be higher were the two tests perfectly reliable. A correction for attenuation of the correlation due to unreliability (Guilford, 1954) estimated the true correlation to be 46 .

\section{Discussion and Subsidiary Experiments}

The present study failed to confirm the strong view of individual differences in fusion. If some people often fuse words, they should also have difficulty with other judgments about the components, such as which component occurred first or which component occurred on which ear. Thus, one would expect accuracy of temporal order judgment to correlate highly with accuracy of word judgments and with ear judgments. There was, in fact, a significant correlation between temporal order and ear judgments, but it was not large: The correlation, even when corrected for attenuation, accounted for only $21 \%$ of the variance in scores. When an attempt at objective measurement of fusion was used (i.e., the ability to discriminate real words from "fused" words), fusion did occur but was relatively rare. Averaged over all subjects, only $6 \%$ errors were made on the task, and the range of individual differences was rather small.

This restricted range on the word judgment task might be a factor in the lack of correlation with the other two tasks. The actual word judgment task may also be inappropriate; that is, some people may commonly fuse the components and perceive the components in addition. The perception of components as well would be sufficient to discriminate real words and fused words.

To evaluate this hypothesis and further explore the nature of temporal order judgments, two new judgment tasks were created. One task, temporal order judgment ear (TOJE), used the same tape as the former temporal order task. However, subjects were asked on the new task to judge which ear received a component 
first, not which phoneme occurred first. If the correlation between temporal order judgment phoneme (the former task: TOJP) and TOJE is high, then performance on TOJP probably reflects the ability to make temporal order judgments and not only fusion. The second new task, called Word Judgment 2 (WJ2), was a modification of the earlier word task. Half of the 44-item pairs were components only, as before. The other half of the pairs consisted of an actual word (e.g., BLANKET) presented to one ear and a component (e.g., either LANKET or BANKET) presented to the other ear. Subjects were asked to judge whether no word was present or a word as well as a component was present. As before, subjects had checklists with the possible items before them.

Thirty-one of the previous 36 subjects were obtained for two more sessions and run as described for the main experiment on the two new tasks. The TOJE task was slightly easier than the former TOJP task, but the distribution of subject scores was rather similar. The correlation of the percent correct on the two TOJ tasks was .62 (.71 corrected for attenuation). This result suggests that poor performance on TOJP is determined to a large extent by poor discriminability on temporal order per se, rather than by fusion.

Subjects made more errors on WJ2 than on the previous word judgment task, and the scores were distributed over a broader range. However, the new task correlated more poorly with the old tasks than did the former word judgment task.

These results in their entirety suggest, therefore, that the occurrence of fusion does not prevent some individuals from also making some discriminations based on the components. The results augment those of Poltrock and Hunt (1977), who found fusion rates to correlate significantly with the bias to report stop consonants as leading liquid consonants but not to correlate with a discriminability measure of temporal order judgments.

How are these results to be reconciled with Day's work? Day found that TOJP was related to the number of fusion responses when subjects wrote down whatever they heard, words or components or both. On the other hand, TOJP was not related to TOJE. We found just the opposite on both aspects. The different results, we suggest, reflect different measures. Day's measure of temporal order judgment (percent correct only on liquid-led items) reflects to a very large degree bias to say that the stop consonants lead. Subjects that are completely unaware of the actual nature of the stimuli may be very susceptible to bias; that is, if they think words really are present, they will both report fusions and commonly be wrong on temporal judgments of liquid-led pairs. This bias would not operate on TOJE judgments, so TOJP and TOJE would not correlate when bias strongly affects the chosen TOJP measure. In our study, we used the overall percent correct, a measure less susceptible to bias, as shown by an extremely high correlation with $\mathrm{d}^{\prime}$.

There are other differences between the Day studies and the present one. The stimulus tapes use different words, they are prepared differently, and the exact onset asynchronies of the members of a pair differ. Our subjects were told the nature of the stimuli and made forced-choice judgments on checklists; Day's subjects did not know the exact nature of the stimuli or what stimulus would occur next. These differences might be critical, and we explored them thoroughly. We do not report the experiment fully here, because the potential problems were all allayed.

First, we compared performance on our tapes with performance on tapes prepared in the Poltrock-Hunt laboratory and kindly lent to us by them. Whereas our tapes were constructed by hand from natural language samples, their tapes used computer-digitized and synchronized speech samples. Nonetheless, subjects' total errors of temporal order judgment on our tape correlated 1.0 (when corrected for attenuation due to unreliability) with performance on their tape. Thus, idiosyncracies of the tapes seem not important.

Second, we ran subjects in an initial session using a procedure similar to Day's. We told subjects nothing about the nature of the stimuli and did not provide them with a checklist. Instead, subjects were told simply to report whatever they had heard, whether words or nonsense words. In a second session, subjects were told the nature of the stimuli, that is, that no words were actually present. They were given a checklist and told to make temporal order judgments of phonemes, just as in the first experiment. We found subjects' discriminability performance (total percent correct) to correlate .98 across the two sessions when corrected for attenuation. Thus, a measure of discriminability taps the same individual difference regardless of test method. On the other hand, a measure of bias (percent correct on stop-led pairs minus percent correct on liquid-led pairs) correlated .68 across sessions when corrected for attenuation. As expected, bias is considerably more susceptible to subjects' expectations than is discriminability. These results justify, therefore, the separation of perceptual judgments into the components of discriminability and bias.

The results of these experiments force us to adopt a weaker view of the nature of individual differences in fusion. We reject the notion that many individuals are "language bound" in the sense that they cannot access information about the word components when the task calls for it. However, there still may be individual differences in the extent to which linguistic information biases perception. We now turn to a discussion of several possible mechanisms by which this bias could operate to produce fusions.

EXPERIMENT 2:

\section{THEORETICAL INVESTIGATIONS OF FUSION}

What are the processing mechanisms that underlie the presence or absence of fusion? We investigated a 
variety of hypotheses, most of which received little experimental support. These will each be mentioned briefly, together with a description of the nature of the evidence that made them untenable. Then we will examine in detail two hypotheses that fared somewhat better.

Poltrock and Hunt (1977) suggested that fusion may result when subjects localize a phoneme in the wrong ear. For example, if BACK is presented to the left ear and LACK to the right, and if the $\mathrm{L}$ is mistakenly localized to the left, then among other things, the subject may report BLACK, a fusion response. But the localization hypothesis predicts that the opposite will sometimes occur; that is, a word may separate into components. Thus, if BLACK is presented to the left ear and LACK to the right, the L in BLACK may be mistakenly localized on the left, yielding a perception of BACK and LACK. Poltrock and Hunt rejected the localization hypothesis because the predicted correlation between fusion rate and separation rate did not occur. Results from our first experiment also are not consistent with the hypothesis. If fusion results from localization difficulties, then difficulty in distinguishing words from nonwords should correlate with ability to localize which component appears in the right ear. It did not.

Originally, a levels-of-processing hypothesis motivated this series of studies. Some people were thought to attend rather peripherally, selecting some messages for further analysis after sensory processing. Thus, if a different message were received by each ear, one message would be filtered prior to semantic or other memorial analysis. Filtering one message would prevent fusion. This notion is similar to Broadbent's (1958) early filter theory. Other people were thought to attend at a higher level of processing, selecting only after sensory inputs had contacted their memorial representatives. Fusion would be likely because all phonemes that define a word would be processed and would be in nearly the correct order. This mode of processing is related to the notions of Deutsch and Deutsch (1963), Keele (1973), and others that postulate parallel access of signals to memorial structures. Broadbent's (1971) and Treisman's (1964) concepts of attenuation provide a bridge between the two views. If one message is attenuated while another is fully processed, then individuals might vary on a continuum from strict filtering to unlimited parallel access to memory, depending on the degree of attenuation.

A strong levels hypothesis is effectively ruled out by Experiment 1. If some people always attend more centrally than others, then the three measures of fusiontemporal order judgment, ear judgment, and word judgment-should all correlate highly with each other. But they do not. Apparently, people have access to knowledge from both "levels."

In recent years, theoretical conceptions of attention have shifted from a levels conception to one of inde- pendent codes that are accessed in parallel (Keele \& Neill, 1978; Posner, 1978). A third hypothesis about why some people have difficulty in temporal order judgment of the two components derives from a conception of coexistent codes. Although the phonemic components are available for perception, the word code is also available, and it interferes with judgment. If only the input phonemes were perceived, perhaps temporal judgments could be made, but a different phonemic ordering is implied by perception of the word, and if the word code is not clearly kept separate from the phonemic code, interference occurs. In its general form, this hypothesis states that some people have more difficulty than others in keeping highly related codes separated.

Three tasks were used to test the code-separation hypothesis. Again, because evidence for the hypothesis turned out negative, the tasks and results will only be briefly described. A fourth task in the same series, designed to explore a somewhat different hypothesis, did correlate with the temporal order judgment task.

The first task was built on a paradigm first studied by Posner and Mitchell (1967). Subjects responded as to whether two successively presented letters were physically the same (e.g., A A). There were two kinds of "different" trials, those in which the name was the same even though the physical form of the letters differed (e.g., $A$ a) and those in which the letter also differed (e.g., A T). One letter followed the other by $500 \mathrm{msec}$. In such a situation, this physical code from the first letter has decayed to some extent, and name information appears as readily available as physical information, providing an opportunity for name interference on physical judgments. It was predicted that people relatively poor on making temporal order judgments would show relatively larger reaction times to respond "different" when a pair of physically different letters had the same name than when they had different names, because both temporal order judgment and same-name interference would reflect difficulty in ignoring the conflict-producing code.

The second task in essence was the reverse of the first. Subjects responded that simultaneous letter pairs were the same if the letters had the same name (e.g., $\mathrm{A} \mathrm{a}$ ), and, in fact, there were no physically identical pairs. Members of "different" pairs were either similar in shape (e.g., E F) or dissimilar in shape (e.g., A T). It was expected that, again, subjects who had difficulty on temporal order judgments would this time show greater difficulty in ignoring physical similarity.

Third, a phoneme-matching task was adopted from Hanson (1977). Subjects were presented pairs of $/ \mathrm{ba} /$ and $/ \mathrm{pa} /$ sounds that varied in discrete 5-msec steps on voice-onset time. For each subject, the boundary voiceonset time that separated a /ba/from a /pa/ sound was determined. Then, in the matching phase of the study, they were told to respond that two sounds were different if they differed at all in sound, even when they were 
members of the same phoneme category. It was expected that people poor on making temporal order judgments would make relatively more errors of responding "same" to two phonemes from the same category that, in fact, were different.

Finally, a fifth task was included because it fit well with the general series, but it is analytic to a different hypothesis. Subjects matched simultaneous letter pairs as the same if they either were physically the same (e.g., A A) or had the same name (e.g., A a). The smaller the reaction time difference between these two conditions, the faster access one has to the name code relative to the physical code. If people who perform poorly on temporal order judgments of word components do so because the word that the components belong to is rapidly available, then it might be expected that a small name-match minus physical-match difference would lead to poor temporal order judgment.

The results provided no support for the codeseparation hypothesis. The correlations between temporal order judgment and the various measures of code interference were uniformly not significant.

The final task of name-match time minus physicalmatch time, which was designed to test another hypothesis, did correlate about .60 with discriminability measures of temporal order judgment. That task reflects the relative speed of access to name codes, and prior expectations were that people with fast access to names would show more interference from a name code on temporal order judgments of phonemes. However, the direction of correlation was the opposite of that expected: People with fast access to letter names tended to be better at discriminating temporal order of phonemes on the Day task.

Why might people with relatively fast access to name codes (relative to physical codes) be better at the temporal order judgment of phonemes on the Day task? One possible hypothesis is related to conceptions of hemispheric specialization. Some evidence suggests that, in most (right-handed) people, the left hemisphere is specialized for temporal order judgment (Carmon \& Nachson, 1971; Efron, 1963). Since the left hemisphere is also the dominant hemisphere for language, performance on temporal order tasks such as Day's phonemic judgment task and on language tasks in general might correlate by virtue of a common hemispheric origin. A version of this hypothesis is that perception and production of spoken language requires good temporal order acuity. Therefore, language ability has as a precursor the ability to make temporal order judgments. An assumption underlying this view is that Day's task involving temporal judgments of phonemes taps ability in making temporal order judgments in general and not just for phonemes; the fact that the stimuli contain phonemes is incidental.

To this point, our hypotheses regarding the fusion phenomenon had been dominated by a suspicion that it depended on language. We found no evidence that that was the case. Our final study in this series, therefore, was designed primarily to determine whether temporal order judgment of phonemes is related to judgments of auditory temporal order independently of verbal content. Recall that in Experiment 1 the phoneme judgment task correlated quite highly with temporal judgments regarding which ear was first stimulated. In this experiment, performance on temporal order judgments of phonemes was correlated with performance on temporal order judgments of tones.

In addition, this experiment sought to replicate the relation found earlier between relative name-retrieval time in letter matching and temporal order performance.

\section{Method}

Subjects. Twenty-four subjects, whose native language was English, were drawn from the University of Oregon paid subject pool. All claimed to be right-handed, to have normal hearing, and to have normal or corrected-to-normal vision.

Tasks and Procedure. Subjects were run on three tasks on each of two sessions. The first task was the TOJP previously described. Components derived from words were presented with the component to one ear leading the component to the other ear by $80 \mathrm{msec}$. Half of the time, the stop component led; half of the time, the liquid component led. Half of the time, the leading component went to the right ear; half of the time, it went to the left. The basic list of 44 components was repeated three times (with differing orders each time) on each of the two sessions. Subjects were provided a checklist of the component pairs in the order of their occurrence. After each presentation of component pairs, subjects checked the one they thought had led.

The second task was a variation of the letter-match task previously described. A pair of letters was simultaneously exposed on an oscilloscope screen. One response key was pressed if the letters had the same name, and another was pressed if they had different names. Half the trials were same-name trials, and half were different-name trials. Within the same-name trials, half the pairs were identical in name only (e.g., A a) and half were also physically identical (e.g., A A).

In the first session, subjects were given three practice blocks of 72 trials each, and then eight data blocks of 72 trials each. On the second session, they had one practice block and eight data blocks, all with 72 trials per block. Subjects initiated a block with a keypress. Then, a new pair of letters appeared immediately after each response. (When two subjects were run together, the new pair appeared after the second response.) At the end of each block, the individual's mean response time and number of trials correct for that block appeared. Finally, a ready signal indicated that subjects could initiate a new block. They were instructed to work as rapidly as possible while maintaining a 95\% accuracy level (68-69 correct).

The third task involved the temporal order judgment of tones. The task was designed, constructed, and presented to subjects so as to be as much as possible like the TOJP task. Pairs of tones, $500.5 \mathrm{~Hz}$ and $1,200.5 \mathrm{~Hz}$, were computer generated with an $80-\mathrm{msec}$ :stimulus asynchrony and simultaneous offset $800 \mathrm{msec}$ after the onset of the leading tone. The frequency of the leading tone (high or low) and the ear to which it was presented (left or right) were randomized with replacement. A tape recording was then made of this output. It consisted of a practice block of 10 trials followed by four individually randomized blocks of 72 trials each. There was a pause of $4 \mathrm{sec}$ between trials and a pause of $1 \mathrm{~min}$ between blocks. 
Subjects were provided response sheets similar in format to those used in the first task. Instead of component pairs, each sheet had columns of " $H$ " and " $L$ " for high and low. After each presentation of tone pairs, subjects checked the letter corresponding to the leading tone. The same tape was presented at both sessions.

The three tasks were scheduled over a 4-day period, with each task being run in two sessions of $30 \mathrm{~min}$ to $1 \mathrm{~h}$ each. Typically, subjects were run in groups of four on the phoneme task, with each subject having separate earphones, and in groups of two on the other tasks. On the matching task, the two subjects were in separate rooms; in the tone task, the subjects had separate earphones and were divided by a small visual screen.

\section{Results}

Table 2 shows the intercorrelations between selected measures from the three tasks. The major diagonal presents the reliabilities of the measures as derived from the correlations between the two sessions. Two measures were used for temporal order judgment of phonemes: One measure was the error averaged over both stop-led and liquid-led pairs, which is a measure of discriminability; the other measure was percent correct on stop-led pairs minus percent correct on liquid-led pairs, divided by the total percent correct, which yields a measure of bias indicating that stops lead. ${ }^{1}$ The measure selected for the matching task was correct reaction times to pairs of letters that match in name minus the reaction time to match physically identical letters. The measure for the tone task was overall percent correct. Also given in Table 2 are the estimated correlations between the measures when corrected for attenuation due to unreliability. Uncorrected correlations of .34 or larger were significant at the .05 level of confidence (one-tailed test).

Temporal order performance on phonemes correlated significantly with temporal order performance on tones (.62, corrected for attenuation), suggesting they are based in part on a common ability. Both of those tasks showed only a small correlation with the matching task, however, and although they were in the same direction as in the last study, they were not significant at the .05 level of confidence even by a one-tailed test.

\section{Discussion}

Experiment 2 provides additional evidence that judgment of phoneme order reflects temporal order ability regardless of language content. Judgment of tone order, which has no apparent language aspect, correlates quite highly with judgment of phoneme order, which is embedded in a language context.

In turn, these results imply that studies that relate judgments of phoneme order to other cognitive abilities are probably examining the relation of temporal order acuity and not language boundness to those other abilities. One reason that temporal order acuity may be related to other language tasks is that greater sensitivity to temporal order could possibly aid in an individual's language development. Whether individual differences in auditory temporal order acuity generalize to other modalities remains to be determined.

One might argue that the two temporal order tasks in the present experiment correlated only about .62 , leaving over $60 \%$ of the variance unaccounted for. Indeed, this does provide leeway for the order-ofphoneme judgments to be influenced by something like language boundness. However, our exploratory attempts to evaluate the levels hypothesis and the code-separation hypothesis did not support such a view. Those attempts included manipulations that could be construed as examining the effects of higher order language properties on lower order judgments. For example, name identity interferes with judgment of physical difference both in visual and in auditory letter matching, but these "language" interference effects fail to correlate appreciably with each other or with temporal order judgments of phonemes.

The less-than-perfect correlation between the two temporal order tasks quite plausibly reflects factors other than language involvement in the one case. Since the stimuli are different, there is no reason to suspect that a person's resolving power of temporal order would be exactly the same for different kinds of stimuli. The judgment of phoneme order might not correlate more highly with judgment of tone order even if the phonemes were removed from the context of word components.

Some mention must be made of the low (and nonsignificant) correlations between relative speed of access to letter names in a letter-matching paradigm and ability on the two temporal order tasks. These results do not replicate the exploratory study preceding Experiment 2. On the other hand, the results do not contradict the exploratory study. The letter-matching task

Table 2

Intercorrelations (r) of Letter Matching Temporal Order Judgment of Phonemes and of Tones

\begin{tabular}{|c|c|c|c|c|c|c|c|c|c|c|}
\hline & \multicolumn{3}{|c|}{ Phoneme Discriminability S+L } & \multicolumn{3}{|c|}{ Phoneme Bias S-L/S+L } & \multicolumn{3}{|c|}{ Match NI-PI } & \multirow{2}{*}{$\begin{array}{l}\text { Tone Per- } \\
\text { cent Cor- } \\
\text { rect (R) }\end{array}$} \\
\hline & $\mathbf{R}$ & $r$ & $\mathrm{I}^{*}$ & $\mathrm{R}$ & $r$ & $\mathrm{r}^{*}$ & $\mathbf{R}$ & $r$ & $r^{*}$ & \\
\hline $\begin{array}{l}\mathrm{S}+\mathrm{L} \\
\mathrm{S}-\mathrm{L} / \mathrm{S}+\mathrm{L} \\
\mathrm{NI}-\mathrm{PI} \\
\text { Tone }\end{array}$ & .85 & $\begin{array}{r}-.59 \\
-.31 \\
.52\end{array}$ & $\begin{array}{r}-.70 \\
-.41 \\
.62\end{array}$ & .84 & $\begin{array}{r}.26 \\
-.31\end{array}$ & $\begin{array}{l}.34 \\
.44\end{array}$ & .70 & -.33 & -.42 & .84 \\
\hline
\end{tabular}

Note $-R=$ reliability.

"Corrected for attenuation. 
yielded poor reliability in the present study, and the correlations with the other tasks, although low and nonsignificant, were at least in the same direction as in the exploratory study. Moreover, the obtained correlations were well within the $95 \%$ confidence bounds of what would be expected were the true correlations approximately what was found in the earlier study. Unfortunately, with small subject samples (e.g., 20 to 30), even moderately high correlations (e.g., .60) may not replicate due simply to statistical power. Finally, one might not expect letter access time to be highly related to temporal order acuity, as the task of letter matching appears to involve little in the way of temporal order. The problem requires further study.

\section{GENERAL DISCUSSION}

The results of our experiments were, on the whole, rather discouraging for the hypothesis that individual differences in fusion are caused by differences in the influence of linguistic rules on perception. Experiment 1 showed that there are no general, cross-task individual differences in the ability to make various judgments about the word components. According to what we take to be the original meaning of fusion, fusers should have been very poor at making such judgments in each of our tasks.

The primary end product of these investigations, therefore, is to alter the direction of search for fundamental information processes that may underlie a variety of language-related individual differences. Fusion of speech presented to the two ears appears not to be fundamental. What we uncovered instead were robust individual differences in ability to make temporal order judgments. Experiment 2 demonstrated that this ability is not restricted to linguistic stimuli.

We do not wish to argue that weaker forms of the fusion hypothesis are nuled out by our studies. For example, it is still possible that people differ in their bias to hear words when given word components. However, we were unable to verify any of several effects we expected from various mechanisms for producing this bias.

According to our current view, a prototypical "fuser" would be an individual who tends to have difficulty discriminating the temporal order of the word components and, also, perhaps has a bias to decide that poorly resolved word-like stimuli were probably words. Of these two components of fusion, we view temporal order discrimination ability as the more interesting. This ability could possibly be fundamental to a variety of cognitive tasks, including speech perception itself.

\section{REFERENCE NOTES}

1. Day, R. S. Temporal order judgments in speech: Are individuals language-bound or stimulus-bound? (Status Report on Speech Research SR-21/22). New Haven, Conn: Haskins Laboratories, 1970.

2. Day, R. S. Digit-span memory in language-bound and stimulus-bound subjects (Status Report on Speech Research SR-34). New Haven, Conn: Haskins Laboratories, 1973.

3. Day, R. S. On learning "secret languages" (Status Report on Speech Research SR-34). New Haven, Conn: Haskins Laboratories, 1973.

\section{REFERENCES}

Broadbent, D. E. Perception and communication. New York: Pergamon, 1958.

Broadbent, D. E. Decision and stress. London: Academic Press, 1971.

Carmon, A., \& Nachson, I. Effect of unilateral brain damage on perception of temporal order. Cortex, 1971, 1, 410-418.

CutTing, J. E. Aspects of phonological fusion. Journal of Experimental Psychology: Human Perception and Performance, 1975, 104, 105-120.

Deutsch, J. A., \& Deutsch, D. Attention: Some theoretical considerations. Psychological Review, 1963, 70, 80-90.

Erron, R. The effect of handedness on the perception of simultaneity and temporal order. Brain, 1963, 86, 261-284.

GOPHER, D. Eye-movement patterns in selective listening tasks of focused attention. Perception \& Psychophysics, 1973, 14, 259 264.

Guilfond, J. P. Psychometric methods. New York: McGrawHill, 1954.

HaNson, V. L. Within-category discriminations in speech perception. Perception \& Psychophysics, 1977, 21, 423-430.

Keele, S. W. Attention and human performance. Pacific Palisades, Calif: Goodyear, 1973.

Keele, S. W., \& Boies, S. J. Processing demands of sequential information. Memory \& Cognition, 1973, 1, 85-90.

Keele, S. W., \& Neill, W. T. Mechanisms of attention. In E. C. Carterette \& M. P. Friedman (Eds.), Handbook of perception (Vol. 9). New York: Academic Press, 1978.

Polthock, S. E., \& Hunt, E. Individual differences in fusion and separation errors. Journal of Experimental Psychology: Human Perception and Performance, 1977, 3, 62-74.

Poswer, M. I. Chronometric explorations of mind. Hillsdale, N.J: Erlbaum, 1978.

Posner, M. I., \& Mitchell, R. F. Chronometric analysis of classification. Psychological Review, 1967, 74, 392-409.

Treisman, A. Monitoring and storage of irrelevant messages in selective attention. Journal of Verbal Learning and Verbal Behavior, 1964, 3, 449-459.

\section{NOTE}

1. In calculating bias, it is useful to adjust for the overall percent correct. In the absence of such a correction, bias may reduce when overall percent correct falls simply because, if a person makes few correct responses, little bias can be exhibited.

(Manuscript received September 1, 1981; revision accepted for publication June 15, 1982.) 